IJMMS 25:12 (2001) 787-800

PII. S016117120100429X

http://ijmms.hindawi.com

(c) Hindawi Publishing Corp.

\title{
ERGODICITY AND ASYMPTOTICALLY ALMOST PERIODIC SOLUTIONS OF SOME DIFFERENTIAL EQUATIONS
}

\author{
CHUANYI ZHANG
}

(Received 20 December 1999)

\begin{abstract}
Using ergodicity of functions, we prove the existence and uniqueness of (asymptotically) almost periodic solution for some nonlinear differential equations. As a consequence, we generalize a Massera's result. A counterexample is given to show that the ergodic condition cannot be dropped.
\end{abstract}

2000 Mathematics Subject Classification. Primary 34C27, 43A60, 37Axx, 28Dxx.

1. Introduction. The asymptotically almost periodic functions were first introduced in $[10,11]$ by Fréchet. In the modern theory of differential (integral) equations, many authors [5, 9, 12, 13, 14, 25] apply the asymptotic property of the functions to determine the existence of almost periodic solutions. Along with the development of such equations as evolution partial differential equations, retarded functional differential equations, and so forth, where the phase spaces are infinite, the theory of Banachvalued (asymptotically) almost periodic functions has been developed [2, 18, 20, 21, $22,23]$. Some techniques in functional analysis and harmonic analysis are applied to such equations, for example, [3, 24] apply spectrum theory to get almost periodic solutions for some linear abstract evolution differential equations.

Let $X$ be a Banach space. In this paper, we apply ergodicity to get asymptotically almost periodic solutions of the following nonlinear differential equation:

$$
\frac{d x}{d t}=A(t, x)+f(t)
$$

where

$$
A: \mathbb{R} \times X \rightarrow X, \quad f: \mathbb{R} \rightarrow X .
$$

What motivates us is recent development of (1.1). For $x, y \in X$, define

$$
[x, y]=\lim _{h \rightarrow 0^{+}} \frac{1}{h}(\|x+h y\|-\|x\|) .
$$

In the case $X=\mathbb{R}^{n},[16]$ assumes the following conditions:

$\left(\mathrm{K}_{1}\right) A(t, x)$ is a continuous mapping;

$\left(\mathrm{K}_{2}\right) f(t)$ is a continuous mapping and $\|f(t)-A(t, 0)\| \leq N$ for all $t \in \mathbb{R}$, where $N>0$;

$\left(\mathrm{K}_{3}\right)$ there exists a function $p \in \mathscr{C}(\mathbb{R})$, the space of bounded continuous functions on $\mathbb{R}$, such that for some positive constants $\delta, \gamma$, and $T_{0}$, the following two properties hold:

(1) $P(t) \leq-\delta$ for $t \in\left(-\infty, t_{0}\right]$,

(2) $\lim _{t \rightarrow \infty} \int_{s}^{t} p(u) d u /(t-s)=-\gamma$ (uniformly for $s>T_{0}$ ); 
$\left(\mathrm{K}_{4}\right)$ for all $(t, x, y) \in \mathbb{R} \times \mathbb{R}^{n} \times \mathbb{R}^{n}$

$$
[x-y, A(t, x)-A(t, y)] \leq p(t)\|x-y\| .
$$

Note that $\left(\mathrm{K}_{4}\right)$ is a strong dissipativity condition.

The main result in [16] is: suppose that conditions $\left(\mathrm{K}_{1}\right)-\left(\mathrm{K}_{4}\right)$ are satisfied. Suppose, furthermore, that $A(t, x)$ is almost periodic in $t$ uniformly for $x \in \mathbb{R}^{n}$ and $f$ is almost periodic. Then equation (1.1) has a unique almost periodic solution on $\mathbb{R}$.

[1] extended the result in [16] by allowing $X$ to be a Banach space and instead of $\left(\mathrm{K}_{3}\right)$, using the following equivalent condition:

$\left(\mathrm{H}_{3}\right)$ there exist a function $p \in \mathscr{C}(\mathbb{R})$ and positive constants $\delta, \delta_{1}, T_{0}$, and $T_{1}$ such that

$$
\begin{aligned}
& p(t) \leq-\delta, \quad\left(t \in\left(-\infty, T_{0}\right]\right) \\
& p(t) \leq-\delta_{1}, \quad\left(t \in\left[T_{1},+\infty\right)\right) .
\end{aligned}
$$

Massera [19] considered the following special case of (1.1) in the case $X=\mathbb{R}^{n}$ :

$$
\frac{d x}{d t}=A(t) x+f(t)
$$

and gave the statement: let the matrix $A(t)$ in (1.6) be real almost periodic such that $a_{i j}=0$ for all $i>j$, then (1.6) has a unique solution for every $f \in \mathscr{C}(\mathbb{R})^{n}$ if and only if the mean value $\lim _{T \rightarrow \infty} \int_{-T}^{T} a_{i i}(t) d t / 2 T \neq 0$ for $1 \leq i \leq n$; in this case, if $f$ is almost periodic, then the unique bounded solution of (1.6) is also almost periodic.

Note that, the Massera's result is not a consequence of $[1,16]$ because in general, $a_{i i}$ do not satisfy $\left(\mathrm{H}_{3}\right)$ or $\left(\mathrm{K}_{3}\right), i=1,2, \ldots, n$. For example, $g(t)=-1 / 2+\sin t$, then $\lim _{T \rightarrow \infty} \int_{-T}^{T} g(t) d t / 2 T=-1 / 2$, but obviously $g$ does not satisfy either $\left(\mathrm{H}_{3}\right)$ or $\left(\mathrm{K}_{3}\right)$.

In this paper, we unify all the results above by ergodicity (for the definition, see Definition 3.1). In Section 2, we show some results on asymptotically almost periodic functions. We then apply ergodicity to investigate asymptotically almost periodic solutions of (1.1) in Section 3. $\left(\mathrm{K}_{3}\right)$ and $\left(\mathrm{H}_{3}\right)$ are regarded novel assumptions in [16] and [1], respectively. We point out that both conditions $\left(\mathrm{K}_{3}\right)$ and $\left(\mathrm{H}_{3}\right)$ are special cases of ergodicity. At the same time, we also point out that Massera's result does not depend on the almost periodicity, but the ergodicity. The almost periodicity is also a special case of ergodicity. Thus, as a corollary we get result of [1] in Section 3 and generalize the result of [19] in Section 4, respectively. Finally, a counterexample is given to show that the ergodic condition cannot be dropped.

2. Vector-valued asymptotically almost periodic functions. In this section, we present some results on asymptotically almost periodic functions. We apply the results to get asymptotically almost periodic solutions in Section 3 .

Let $\Omega$ be a closed subset of $X$, let $J \in\left\{\mathbb{R}^{+}, \mathbb{R}\right\}$, and let $\mathscr{C}(J \times \Omega, X)$ (respectively, $\mathscr{C}(J, X)$ ) be the space of bounded, continuous functions from $J \times \Omega$ (respectively, $J$ ) to $X$ with supremum norm. When $X=\mathbb{C}$, we will omit $X$ in our notations. For example, we write $\mathscr{C}(\mathbb{R})$ for $\mathscr{C}(\mathbb{R}, \mathbb{C})$. 
Definition 2.1. A subset $P$ of $J$ is said to be relatively dense in $J$ if there exists a number $l>0$ such that

$$
[t, t+l] \cap P \neq \varnothing \quad(t \in J) .
$$

DEFinItion 2.2. A function $f \in \mathscr{b}(\mathbb{R} \times \Omega, X)$ is said to be almost periodic in $t \in \mathbb{R}$ and uniform on compact subsets of $\Omega$ if for every $\epsilon>0$ and every compact subset $K$ of $\Omega$, there exists a relatively dense subset $P$ of $\mathbb{R}$ such that

$$
\|f(t+\tau, x)-f(t, x)\|<\epsilon \quad(\tau \in P, t \in \mathbb{R}, x \in K) .
$$

Denote by $\mathscr{A} \mathscr{P}(\mathbb{R} \times \Omega, X)$ all such functions.

For $f \in \mathscr{C}(J \times \Omega, X)$ and $s \in J$, the translate of $f$ by $s$ is the function $R_{s} f(t, x)=$ $f(t+s, x), t \in J$ and $x \in \Omega$. Then $f$ is in $\mathscr{A P}(\mathbb{R} \times \Omega, X)$ if and only if $\left\{R_{s} f: s \in \mathbb{R}\right\}$ is relatively compact in $\mathscr{C}(\mathbb{R} \times \Omega, X)$.

DefinItion 2.3. A function $f \in \mathscr{b}(J \times \Omega, X)$ is said to be asymptotically almost periodic in $t \in J$ and uniform on compact subsets of $\Omega$ if for every $\epsilon>0$ and every compact subset $K$ of $\Omega$, there exist a relatively dense subset $P$ and a bounded subset $C$ of $J$ such that

$$
\|f(t+\tau, x)-f(t, x)\|<\epsilon \quad(\tau \in P, t, t+\tau \in J \backslash C, x \in K) .
$$

Denote by $\mathscr{A} \mathscr{A} \mathscr{P}(J \times \Omega, X)$ all such functions.

One sees that the asymptotically almost periodicity will reduce to the almost periodicity if $C=\varnothing$ and $J=\mathbb{R}$.

THEOREM 2.4. An $f \in \mathscr{A} A \mathscr{P}(J \times \Omega, X)$ is uniformly continuous on $J \times K$ and the range $f(J \times K)$ is relatively compact.

Proof. For the case $J=\mathbb{R}^{+}$, this is [22, Lemma 3.2]. For compact $K$, there exists a natural identification between $\mathscr{C}(J \times K, X)$ and $\mathscr{b}(J, \mathscr{C}(K \times X))$; this identifies $\mathscr{A} \mathscr{A} \mathscr{P}(J \times K, X)$ and $\mathscr{A} \mathscr{A} \mathscr{P}(J, \mathscr{C}(K \times X))$. Note that if $f \in \mathscr{A} \mathscr{A} \mathscr{P}(\mathbb{R}, X)$ then $\left.f\right|_{\mathbb{R}^{+}} \in$ $\mathscr{A} \mathscr{A} \mathscr{P}\left(\mathbb{R}^{+}, X\right)$ and $\left.f\right|_{\mathbb{R}^{-}} \in \mathscr{A} \mathscr{A} \mathscr{P}\left(\mathbb{R}^{-}, X\right)$. So the theorem is a consequence of [22, Lemma 3.2].

As usual, $\mathscr{C}_{0}(J \times \Omega, X)$ consists of the functions $f \in \mathscr{C}(J \times \Omega, X)$ that vanish at infinity. That is, for $\epsilon>0$ there exist a bounded subset $C$ of $J$ such that

$$
\|f(t, x)\|<\epsilon, \quad(t \in J \backslash C, x \in K) .
$$

REMARK 2.5. (i) $\mathscr{A} \mathscr{A} \mathscr{P}(J)$ was originally introduced in $[10,11]$ by Fréchet in the case $J=\mathbb{R}^{+}$. It is well known (cf. [22, Theorem 3.4]) that $f \in \mathscr{A} A \mathscr{P}\left(\mathbb{R}^{+}, X\right)$ if and only if $f=\left.g\right|_{\mathbb{R}^{+}}+\varphi$, where $g \in \mathscr{A} \mathscr{P}(\mathbb{R}, X)$ and $\varphi \in \mathscr{C}_{0}\left(\mathbb{R}^{+}, X\right)$.

(ii) There is a difference of notation, as well as of meaning, between $\mathscr{C}_{0}(\mathbb{R}, X)$ here and $C_{0}^{+}(\mathbb{R}, X)$ in [24] by Ruess and Vu. Here, $\varphi \in \mathscr{C}_{0}(\mathbb{R}, X)$ if and only if $\|\varphi(t)\| \rightarrow 0$ as $|t| \rightarrow \infty$; in [24] $\varphi \in C_{0}^{+}(\mathbb{R}, X)$ if and only if $\|\varphi(t)\| \rightarrow 0$ as $t \rightarrow \infty$.

(iii) This brings a difference in defining asymptotically almost periodicity on $\mathbb{R}$ between here and [24]. In [24], $f$ is asymptotically almost periodic on $\mathbb{R}$ if and only 
if $f=g+\varphi$, where $g \in \mathscr{A} \mathscr{P}(\mathbb{R}, X)$ and $\varphi \in C_{0}^{+}(\mathbb{R}, X)$; the function $f$ will lose some properties (e.g., ergodicity) that an asymptotically almost periodic function on $\mathbb{R}^{+}$has. However, if $g \in \mathscr{A} \mathscr{P}(\mathbb{R} \times \Omega, X)$ and $\varphi \in \mathscr{C}_{0}(\mathbb{R} \times \Omega, X)$, then the function $f=g+\varphi$ satisfies the conditions in Definition 2.3; conversely Theorem 2.6 states that the function in Definition 2.3 will have a unique such decomposition. Definition 2.3 is more natural and consistent.

THEOREM 2.6. A function $f \in \mathscr{b}(J \times \Omega, X)$ is asymptotically almost periodic if and only if there is a unique function $g \in \mathscr{A} \mathscr{P}(\mathbb{R} \times \Omega, X)$ such that $f-\left.g\right|_{J \times K} \in \mathscr{C}_{0}(J \times \Omega, X)$ for every compact subset $K$ of $\Omega$.

For $J=\mathbb{R}^{+}$this is [22, Theorem 3.4]. For the general case, see [28, Theorem 11 and Remark 12(2)].

Lemma 2.7 generalizes [7, Theorem 2.8] from finite-dimensional space $\mathbb{C}^{n}$ to a Banach space. The proof is similar; so we omit it.

LEMMA 2.7. If $g \in \mathscr{A} \mathscr{P}(\mathbb{R} \times \Omega, X)$ and $G \in \mathscr{A} \mathscr{P}(\mathbb{R}, \Omega)$, then the composition $g(\cdot, G(\cdot))$ is in $\mathscr{A P}(\mathbb{R}, X)$.

THEOREM 2.8. If $f \in \mathscr{A} \mathscr{A} \mathscr{P}(J \times \Omega, X)$ and $F \in \mathscr{A} \mathscr{A} \mathscr{P}(J, \Omega)$, then the composition $f(\cdot, F(\cdot))$ is in $\mathscr{A} \mathscr{A} \mathscr{P}(J, X)$.

Proof. We show the case $J=\mathbb{R}$ only. Similarly, one shows the case $J=\mathbb{R}^{+}$. Since $F(J)$ is relatively compact, we may assume that $\Omega$ is compact. By assumptions,

$$
f=g+\varphi, \quad F=G+\Phi,
$$

where $g \in \mathscr{A} \mathscr{P}(\mathbb{R} \times \Omega, X), G \in \mathscr{A} \mathscr{P}(\mathbb{R}, X), \varphi \in \mathscr{C}_{0}(\mathbb{R} \times \Omega, X)$, and $\Phi \in \mathscr{C}_{0}(\mathbb{R}, X)$. So

$$
\begin{aligned}
f(t, F(t)) & =g(t, G(t))+[f(t, F(t))-g(t, G(t))] \\
& =g(t, G(t))+[g(t, F(t))-g(t, G(t))+\varphi(t, F(t))] .
\end{aligned}
$$

By Lemma $2.7, g(t, G(t)) \in \mathscr{A} \mathscr{P}(\mathbb{R}, X)$. Obviously $\varphi(t, F(t)) \in \mathscr{C}_{0}(\mathbb{R}, X)$. To prove the theorem, we need to show that the function $g(t, F(t))-g(t, G(t))$ is in $\mathscr{C}_{0}(\mathbb{R}, X)$. Note that $\overline{F(\mathbb{R})} \supset G(\mathbb{R})$ (see [27, Lemma 1.3] or [26]). Since $g$ is uniformly continuous on $\mathbb{R} \times \Omega$ and $\Phi \in \mathscr{C}_{0}(\mathbb{R}, X)$, for $\epsilon>0$ there exists $T>0$ such that

$$
\|g(t, F(t))-g(t, G(t))\|<\epsilon \quad(|t|>T) .
$$

The proof is complete.

LEMMA 2.9. Suppose that both $f$ and $f^{\prime}$ are in $\mathscr{A} \mathscr{A P}(J, X)$. That is, $f=g+\varphi$ and $f^{\prime}=\alpha+\beta$ with $g, \alpha \in \mathscr{A} \mathscr{P}(\mathbb{R}, X)$ and $\varphi, \beta \in \mathscr{C}_{0}(J, X)$. Then $g$ and $\varphi$ are differentiable so that

$$
g^{\prime}=\alpha, \quad \varphi^{\prime}=\beta
$$

For the proof, see [9, Theorem 9.2].

As a consequence of Theorem 2.8 and Lemma 2.9, one gets the following theorem. 


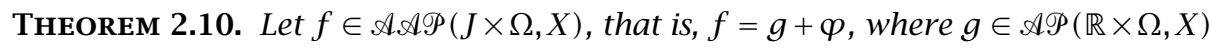
and $\varphi \in \mathscr{C}_{0}(J \times \Omega, X)$. Consider the following equations:

$$
\begin{aligned}
& \frac{d x}{d t}=f(t, x), \\
& \frac{d y}{d t}=g(t, y) .
\end{aligned}
$$

If (2.9) has an asymptotically almost periodic solution $F$, then the almost periodic component of $F$ is a solution of (2.10).

3. Ergodicity and solutions of (1.1). The ergodicity of a scalar function was discussed in [8]. The ergodicity of a vector-valued function was defined in [4]. Now we present it in the following definition.

DefinITION 3.1. A function $f \in \mathscr{C}(J)$ is said to be ergodic if there exists a number $M(f) \in \mathbb{C}$ (is called mean of $f$ ) such that

$$
\lim _{T \rightarrow \infty} \frac{1}{T-a} \int_{a}^{T} f(t+s) d t=M(f)
$$

uniformly with respect to $s \in \mathbb{R}$, where $a=0$ when $J=\mathbb{R}^{+}$and $a=-T$ when $J=\mathbb{R}$.

An ergodic function in Eberlein's meaning [8] and Basit's meaning [4] is required to be uniformly continuous on $J$. But we do not need the requirement here.

Many function spaces are ergodic, for example, the space of almost periodic functions $\mathscr{A} \mathscr{P}(\mathbb{R})$ [7], the space of asymptotically almost periodic functions $\mathscr{A} A \mathscr{P}(\mathbb{R})[8]$, and the space of weakly almost periodic functions $\mathscr{W} \mathscr{A} \mathscr{P}(\mathbb{R})[8]$.

Note that (3.1) is equivalent to

$$
\lim _{T \rightarrow \infty} \frac{1}{T-a} \int_{a+s}^{T+s} f(t) d t=M(f)
$$

uniformly with respect to $s \in J$. That is, for $\epsilon>0$ there exists $T>0$ such that whenever $a, b \in J$ and $b-a>T$, then

$$
\left|\frac{1}{b-a} \int_{a}^{b} f(t) d t-M(f)\right|<\epsilon .
$$

As we point out in the introduction that $\left(\mathrm{K}_{3}\right)$ and $\left(\mathrm{H}_{3}\right)$ are all special cases of ergodicity. For, let $p \in\left(\mathrm{H}_{3}\right)$ and let $-\gamma=\max \left\{-\delta,-\delta_{1}\right\}$ then $p(t) \leq-\gamma$ for all $t \in \mathbb{R}$ except a finite interval. Therefore, there exist a real ergodic function $p_{1}$ with $M\left(p_{1}\right)=-\gamma$ such that $p(t) \leq p_{1}(t)$ for all $t \in \mathbb{R}$. So the following condition is weaker than $\left(\mathrm{H}_{3}\right)$.

$\left(\mathrm{H}_{3}^{\prime}\right)$ There exists a real ergodic function $p$ with $M(p)=-\gamma<0$.

LEMMA 3.2. Let $p$ be real, ergodic with $M(p) \neq 0$. Then the following defined functions are bounded:

(1) in the case $M(p)<-\gamma<0$

$$
\begin{gathered}
g_{1}(t)=\int_{a}^{t} e^{\int_{s}^{t} p(u) d u} d s \quad(t \in J), \\
g_{2}(t, s)=e^{\int_{s}^{t} p(r) d r} \quad(t, s \in \mathbb{R}, t \geq s),
\end{gathered}
$$


(2) in the case $M(p)>\gamma>0$

$$
g(t)=-\int_{t}^{\infty} e^{\int_{s}^{t} p(u) d u} d s \quad(t \in J)
$$

where $a=0$ when $J=\mathbb{R}^{+}$and $a=-\infty$ when $J=\mathbb{R}$.

Proof. We show (3.4) for the case $J=\mathbb{R}^{+}$only. Similarly, one shows the lemma for other cases.

Since $p$ is ergodic with $M(p)<-\gamma<0$, by (3.3) there exist $T>0$ such that for any $a, b \in \mathbb{R}^{+}, b-a>T$, we have

$$
\int_{a}^{b} p(u) d u<-r(b-a) .
$$

For $t \in \mathbb{R}^{+}$there is a positive integer $n$ such that $(n-1) T<t \leq n T$. Let $B=\exp \{\|p\| T\}$. Then

$$
\begin{aligned}
g(t) & =\int_{0}^{t} e^{\int_{s}^{t} p(u) d u} d s \leq \int_{0}^{n T} e^{\int_{s}^{t} p(u) d u} d s \\
& =\int_{0}^{n T} e^{\int_{s}^{n T}-\int_{t}^{n T} p(u) d u} d s \leq \int_{0}^{n T} e^{\int_{s}^{n T} p(u) d u+\|p\| T} d s \\
& =B\left[\sum_{i=1}^{n-1} \int_{(i-1) T}^{i T}+\int_{(n-1) T}^{n T} e^{\int_{s}^{n T} p(u) d u} d s\right] \\
& \leq B\left[\sum_{i=1}^{n-1} \int_{(i-1) T}^{i T} e^{-\gamma(n T-s)} d s+\int_{(n-1) T}^{n T} e^{\|p\| T} d s\right] \\
& \leq B\left[\sum_{i=1}^{n-1} T e^{-\gamma(n-i) T}+T B\right] \leq B T\left[\sum_{i=1}^{\infty} e^{-r i T}+B\right]<\infty .
\end{aligned}
$$

The proof is complete.

As the proof of Lemma 2.3 in [16], one shows the following lemma.

LEMMA 3.3. Suppose that $\left(K_{4}\right)$ is satisfied. Let $u$ and $v$ be solutions of (1.1) on an interval $[a, b]$. Then

$$
\|u(t)-v(t)\| \leq\|u(a)-v(a)\| e^{\int_{s}^{t} p(r) d r} \quad \forall t \in[a, b] .
$$

THEOREM 3.4. Suppose that $\left(K_{1}\right),\left(K_{2}\right),\left(H_{3}^{\prime}\right)$, and $\left(K_{4}\right)$ are satisfied. Let

$$
\Gamma=\max \left\{1,\left\|g_{1}\right\|,\left\|g_{2}\right\|\right\},
$$

where $g_{1}$ and $g_{2}$ are as in Lemma 3.2(1). Then (1.1) has a unique bounded solution $u$ on $J$. (In the case $J=\mathbb{R}^{+}$the solution $u$ depends on initial value.) Furthermore, if $v$ is any solution of (1.1), then $\|u(t)-v(t)\| \rightarrow 0$ as $t \rightarrow \infty$.

Proof. We proof for the case $J=\mathbb{R}$, similarly one shows the case of $J=\mathbb{R}^{+}$.

If $A(t, 0) \neq 0$ for $t \in \mathbb{R}$, we replace $A(t, x)$ and $f(t)$ by $A(t, x)-A(t, 0)$ and $f(t)+$ $A(t, 0)$, respectively. We assume, henceforth, that $A(t, 0)=0$ and $f(t) \leq N$ for all $t \in \mathbb{R}$. 
We fix a vector $u_{0} \in X$ and let $\left\|u_{0}\right\|=r_{0}$. For each positive integer $n$ we consider the following Cauchy problem:

$$
x^{\prime}=A(t, x)+f(t), \quad x(-n)=u_{0} .
$$

Then (3.11) has a unique solution $u_{n}$ on $[-n, n]$ (see [15]). We first show that there is $r>0$ such that

$$
\left\|u_{n}\right\| \leq r \quad \forall n
$$

By $\left(\mathrm{K}_{4}\right)$ we have

$$
\begin{aligned}
D_{+}\left\|u_{n}(t)\right\| & =\left[u_{n}(t), u_{n}^{\prime}(t)\right]=\left[u_{n}(t), A\left(t, u_{n}(t)\right)+f(t)\right] \\
& \leq\left[u_{n}(t), A\left(t, u_{n}(t)\right)\right]+\left[u_{n}(t), f(t)\right] \\
& \leq p(t)\left\|u_{n}(t)\right\|+\|f(t)\|,
\end{aligned}
$$

where $D_{+}\|u(t)\|$ denotes the right derivative of $\|u(t)\|$. So

$$
\left\|u_{n}(t)\right\| \leq\left\|u_{n}(-n)\right\| e_{-n}^{t} p(r) d r+\int_{-n}^{t}\|f(t)\| e^{\int_{s}^{t} p(r) d r} d s \leq \Gamma\left(r_{0}+N\right)=r .
$$

It follows that $\left\|u_{n}(t)\right\| \leq r$ for all $t \in[-n, n]$ and for all $n$.

Next, we show that the sequence $\left\{u_{n}\right\}$ is a uniform Cauchy sequence in every bounded subset $[-h, h]$ of $\mathbb{R}$. Indeed, let $n$ and $m$ be two positive integers, $m \geq n$. Then $u_{n}$ and $u_{m}$ are defined on $[-n, n]$. By (3.9) for $t \in[-n, n]$, we have

$$
\left\|u_{m}(t)-u_{n}(t)\right\| \leq\left\|u_{m}(-n)-u_{n}(-n)\right\| e^{\int_{-n}^{t} p(s) d s} \leq 2 r e^{\int_{-n}^{t} p(s) d s} .
$$

Let $\delta<\gamma$. By ergodicity of $p$, when $n$ is sufficiently large, one has

$$
e^{\int_{-n}^{t} p(s) d s} \leq e^{-\delta(t+n)} .
$$

This implies that $\left\{u_{n}\right\}$ is a uniform Cauchy sequence in every bounded subset of $\mathbb{R}$. Thus its limit is a bounded solution of (1.1).

As the proof of [16], one shows the uniqueness of bounded solution.

The last statement is a consequence of Lemma 3.3. The proof is complete.

THEOREM 3.5. Under the assumptions of Theorem 3.4, if $A(t, x) \in \mathscr{A} A \mathscr{P}(J \times \Omega, X)$ and $f \in \mathscr{A} \mathscr{A P}(J, X)$, then the unique bounded solution of (1.1) is also asymptotically almost periodic.

Proof. Theorem 3.4 has shown the existence and uniqueness of bounded solution $u$ for (1.1). We need to show that $u$ is asymptotically almost periodic if $A(t, x) \in$ $\mathscr{A} \mathscr{A} \mathscr{P}(J \times \Omega, X)$ and $f \in \mathscr{A} \mathscr{A} \mathscr{P}(J, X)$.

Let $S=\{u(t): t \in J\}$. First we show that $S$ is relatively compact in $X$. That is, for any sequence $\left\{s_{n}\right\}$ of $J,\left\{u\left(s_{n}\right)\right\}$ has a convergent subsequence. If it does not, then there exists an $\epsilon>0$ such that

$$
\left\|u\left(s_{n}\right)-u\left(s_{m}\right)\right\| \geq \epsilon
$$

for all distinct numbers $n, m$. 
Without loss of generality, we may assume $s_{n} \rightarrow \infty$. For any fixed $T>0$, let $s_{n}=$ $T+\theta_{n}$. Then $\theta_{n} \rightarrow \infty$.

Since $A \in \mathscr{A} \mathscr{A} \mathscr{P}(J \times \Omega, X)$ and $f \in \mathscr{A} \mathscr{A} \mathscr{P}(J, X)$,

$$
A=G+\Phi, \quad f=g+\varphi,
$$

where $G \in \mathscr{A} \mathscr{P}(\mathbb{R} \times \Omega, X), g \in \mathscr{A} \mathscr{P}(\mathbb{R}, X)$ and $\Phi \in \mathscr{C}_{0}(J \times \Omega, X), \varphi \in \mathscr{C}_{0}(J, X)$. Note that the translate set $\left\{R_{s} G: s \in \mathbb{R}\right\}$ and $\left\{R_{s} g: s \in \mathbb{R}\right\}$ are relatively compact. If necessary by taking a subsequence, we may assume that uniformly $R_{\theta_{n}} G \rightarrow B$ on $\mathbb{R} \times K$ and $R_{\theta_{n}} g \rightarrow h$ on $\mathbb{R}$, where $B \in \mathscr{A} \mathscr{P}(\mathbb{R} \times \Omega, X), h \in \mathscr{A} \mathscr{P}(\mathbb{R}, X)$, and $K$ is any compact subset of $\Omega$. Since $\Phi \in \mathscr{C}_{0}(J \times \Omega, X)$ and $\varphi \in \mathscr{C}_{0}(J, X)$, for any finite interval [0,T] we have uniformly $R_{\theta_{n}} \Phi(t, x) \rightarrow 0(0 \leq t \leq T, x \in K)$ and $R_{\theta_{n}} \varphi(t) \rightarrow 0, t \in[0, T]$. Therefore, for $(t, x) \in[0, T] \times K$

$$
R_{\theta_{n}} A(t, x) \longrightarrow B(t, x), \quad R_{\theta_{n}} f(t) \longrightarrow h(t),
$$

uniformly.

Since $u$ is a solution of (1.1), $u_{n}=R_{\theta_{n}} u$ is a solution of

$$
\frac{d y}{d t}=A\left(t+\theta_{n}, y\right)+f\left(t+\theta_{n}\right) .
$$

Since $B$ satisfies the same conditions as $A$, the limit equation

$$
\frac{d z}{d t}=B(t, z)+h(t)
$$

has a unique bounded solution on $J$. Let $u_{0}$ be this solution. Put

$$
v_{n}(t)=\left\|u_{n}(t)-u_{0}(t)\right\|
$$

Note that $D_{+}\|u(t)\|$ exists and

$$
D_{+}\|u(t)\|=\left[u(t), u^{\prime}(t)\right],
$$

where $D_{+}\|u(t)\|$ denotes the right derivative of $\|u(t)\|$ at $t$. Note the following property of the function $[\cdot, \cdot]$

$$
[x, y+z] \leq[x, y]+\|z\| .
$$

Then it follows from $\left(\mathrm{K}_{4}\right)$, (3.6), and (3.9) that

$$
\begin{aligned}
D_{+} v_{n}(t)= & {\left[u_{n}(t)-u_{0}(t), \frac{d}{d t}\left(u_{n}(t)-u_{0}(t)\right)\right] } \\
= & {\left[u_{n}(t)-u_{0}(t), A\left(t+\theta_{n}, u_{n}(t)\right)+f\left(t+\theta_{n}\right)-B\left(t, u_{0}(t)\right)-h(t)\right] } \\
= & {\left[u_{n}(t)-u_{0}(t), A\left(t+\theta_{n}, u_{n}(t)\right)-A\left(t+\theta_{n}, u_{0}(t)\right)\right.} \\
& \left.\quad+A\left(t+\theta_{n}, u_{0}(t)\right)+f\left(t+\theta_{n}\right)-B\left(t, u_{0}(t)\right)-h(t)\right] \\
\leq & {\left[u_{n}(t)-u_{0}(t), A\left(t+\theta_{n}, u_{n}(t)\right)-A\left(t+\theta_{n}, u_{0}(t)\right)\right] } \\
& +\left\|A\left(t+\theta_{n}, u_{0}(t)\right)+f\left(t+\theta_{n}\right)-B\left(t, u_{0}(t)\right)-h(t)\right\| \\
\leq & p(t)\left\|u_{n}(t)-u_{0}(t)\right\|+b_{n}(t),
\end{aligned}
$$


where

$$
b_{n}(t)=\left\|A\left(t+\theta_{n}, u_{0}(t)\right)+f\left(t+\theta_{n}\right)-B\left(t, u_{0}(t)\right)-h(t)\right\| .
$$

Let $b_{n}(T)=\sup \left\{b_{n}(t): t \in[0, T]\right\}$ and $K=\left\{u_{0}(t): t \in[0, T]\right\}$. Then $b_{n}(T) \rightarrow 0$ as $n \rightarrow \infty$ and $K$ is compact. Now,

$$
D_{+} v_{n}(t) \leq p(t) v_{n}(t)+b_{n}(T)
$$

Integrating (3.11), we have, for all $t \in[0, T]$,

$$
v_{n}(t) \leq e^{\int_{0}^{t} p(u) d u} \cdot v_{n}(0)+\int_{0}^{t} e^{\int_{s}^{t} p(u) d u} b_{n}(T) d s .
$$

Since $p$ is ergodic with $M(p)<-\gamma<0$, let $T>0$ be such that

$$
0 \leq e^{\int_{0}^{T} p(u) d u} \cdot v_{n}(0) \leq e^{-\gamma T} \cdot v_{n}(0)<\frac{\epsilon}{4} .
$$

It follows from Lemma 3.2 that for sufficiently large $n$,

$$
\int_{0}^{T} e^{\int_{s}^{T} p(u) d u} b_{n}(T) d s<\frac{\epsilon}{4} .
$$

That is,

$$
v_{n}(T)=\left\|u_{n}(T)-u_{0}(T)\right\|<\frac{\epsilon}{2}
$$

Now

$$
\begin{aligned}
\epsilon & \leq\left\|u\left(s_{n}\right)-u\left(s_{m}\right)\right\| \\
& \leq\left\|u\left(s_{n}\right)-u_{0}(T)\right\|+\left\|u\left(s_{m}\right)-u_{0}(T)\right\| \\
& =\left\|u_{n}(T)-u_{0}(T)\right\|+\left\|u_{m}(T)-u_{0}(T)\right\|<\epsilon .
\end{aligned}
$$

This is a contradiction. This shows that $\left\{u\left(s_{n}\right)\right\}$ has a convergent subsequence. So $S$ is relatively compact in $X$.

Next we show that $u$ is asymptotically almost periodic. By Definition 2.3 for every $\epsilon>0$ there are a relatively dense subset $P$ and a bounded subset $C$ of $J$ such that, for $\tau \in P, t, t+\tau \in J \backslash C$, and $x \in S$,

$$
\|A(t+\tau, x)-A(t, x)+f(t+\tau)-f(t)\| \leq \epsilon .
$$

So

$$
\begin{aligned}
D_{+} & \|u(t+\tau)-u(t)\| \\
= & {[u(t+\tau)-u(t), A(t+\tau, u(t+\tau))-A(t, u(t))+f(t+\tau)-f(t)] } \\
\leq & {[u(t+\tau)-u(t), A(t, u(t+\tau))-A(t, u(t))] } \\
& +\|A(t+\tau, u(t+\tau))-A(t, u(t+\tau))+f(t+\tau)-f(t)\| \\
\leq & p(t)\|u(t+\tau)-u(t)\|+\epsilon .
\end{aligned}
$$


Let $T>0$ be such that $2\|u\| \exp \{-\gamma T\}<\epsilon$ and $[-T, T] \supset C$. Solving this differential inequality we have, for $t>T$,

$$
\begin{aligned}
\|u(t+\tau)-u(t)\| & \leq\|u(t-T+\tau)-u(t-T)\| e^{\int_{t-T}^{t} p(u) d u}+\epsilon \int_{t-T}^{t} e^{\int_{s}^{t} p(u) d u} d s \\
& \leq 2\|u\| e^{\int_{t-T}^{t} p(u) d u}+\epsilon \int_{0}^{t} e^{\int_{s}^{t} p(u) d u} d s \\
& \leq 2\|u\| e^{-r T}+\epsilon\left\|g_{1}\right\| \leq \epsilon\left(1+\left\|g_{1}\right\|\right),
\end{aligned}
$$

where the function $g_{1}$ is defined in Lemma 3.2. Thus in the case $J=\mathbb{R}^{+}$we have already shown that $u$ is asymptotically almost periodic. If $J=\mathbb{R}$. Let $a<-2 T$ and so, $t=a+T<-T$. Then we have

$$
\begin{aligned}
\|u(a+T+\tau)-u(a+T)\| & \leq\|u(a+\tau)-u(a)\| e^{\int_{a}^{a+T} p(u) d u}+\epsilon \int_{a}^{a+T} e^{\int_{s}^{a+T} p(u) d u} d s \\
& \leq 2\|u\| e^{-r T}+\epsilon\left\|g_{1}\right\| \leq \epsilon\left(1+\left\|g_{1}\right\|\right) .
\end{aligned}
$$

That is,

$$
\|u(t+\tau)-u(t)\| \leq \epsilon\left(1+\left\|g_{1}\right\|\right)
$$

for all $t, t+\tau \in \mathbb{R} \backslash[-T, T]$ and $\tau \in P$. The function $u$ is in $\mathscr{A} \mathscr{A} \mathscr{P}(\mathbb{R}, X)$. The proof is complete.

The following corollary is the main result in [1], and is a consequence of Theorems 2.10, 3.4, and 3.5.

COROLlary 3.6. Suppose that $\left(K_{1}\right),\left(K_{2}\right),\left(H_{3}\right)$, and $\left(K_{4}\right)$ are satisfied. Then (1.1) has a unique bounded solution. In this case, if $A(t, x) \in \mathscr{A} \mathscr{P}(\mathbb{R} \times \Omega, X)$ and $f \in \mathscr{A} \mathscr{P}(\mathbb{R}, X)$, then the unique solution is also an almost periodic solution on $\mathbb{R}$.

4. Applications to solutions of (1.6). Before considering equation (1.6), we first consider the following scalar equation:

$$
x^{\prime}(t)=a(t) x+f(t),
$$

where $a$ and $f$ are in $\mathscr{C}(J)$. We claim that (4.1) satisfies $\left(\mathrm{K}_{4}\right)$ where $p=a$. For, since $a$ is bounded on $J$, for sufficient small $h \in \mathbb{R}$ we have $|1+h a(t)|=1+h a(t)$. So

$$
\begin{aligned}
{[x-y, a(t) x-a(t) y]=} & \lim _{h \rightarrow 0^{+}} \frac{1}{h}\{|x-y+h(a(t) x-a(t) y)|-|x-y|\} \\
& =\lim _{h \rightarrow 0^{+}} \frac{1}{h}\{|x-y||1+h a(t)|-|x-y|\}=a(t)|x-y| .
\end{aligned}
$$

That is, condition $\left(\mathrm{K}_{4}\right)$.

LEMMA 4.1. Let $a \in \mathscr{C}(J)$ be real ergodic. Then (4.1) has a unique bounded solution for every $f \in \mathscr{C}(J)$ if and only if $M(a) \neq 0$. In this case, if $a, f \in \mathscr{A A P}(J)$, then the unique solution is also in $\mathscr{A} \mathscr{A P}(J)$. Furthermore, if $a_{1}$ and $g$ are almost periodic components of $a$ and of $f$, respectively, then the equation

$$
x^{\prime}(t)=a_{1}(t) x+g(t)
$$

has a unique almost periodic solution. 
Proof. Sufficiency. In the case $M(a)<0$, this is a consequence of Theorems $2.10,3.4$, and 3.5 . In the case $M(a)>0$, one can check directly that the function

$$
y(t)=-\int_{t}^{\infty} e^{\int_{s}^{t} a(u) d u} f(s) d s \quad(t \in J)
$$

is a solution of (4.1), is bounded by Lemma 3.2, and is asymptotically almost periodic if both $a$ and $f$ are.

NeCessity. By [17, Theorems 3.2 and 4.1] and [6, Proposition 8.2], equation (4.1) satisfies an exponential dichotomy. That is, there are positive numbers $\alpha_{i}$ and $k_{i}$, $i=1,2$ such that either

$$
\left|y_{0}(t) y_{0}^{-1}(s)\right| \leq k_{1} e^{-\alpha_{1}(t-s)} \quad(t \geq s)
$$

or

$$
\left|y_{0}(t) y_{0}^{-1}(s)\right| \leq k_{2} e^{-\alpha_{2}(s-t)} \quad(t \leq s),
$$

where

$$
y_{0}(t)=e^{\int_{0}^{t} a(u) d u}
$$

is the unique solution of the homogeneous equation of $(4.1)$ with $y_{0}(0)=1$. Suppose the former holds. Then

$$
\left|e^{-\int_{s}^{t} a(u) d u}\right| \leq k_{1} e^{-\alpha_{1}(t-s)} \quad(t \geq s) .
$$

Let $\alpha_{1}>\delta>0$ and $\alpha=\alpha_{1}-\delta$. Then

$$
k_{1} e^{-\alpha_{1}(t-s)}=k_{1} e^{-\left(\alpha_{1}-\delta+\delta\right)(t-s)}=k_{1} e^{-\delta(t-s)} e^{-\alpha(t-s)} .
$$

Therefore, there is $T_{0}$ such that $k_{1} e^{-\delta(t-s)} \leq 1$ when $(t-s) \geq T_{0}$. This implies that, when $(t-s) \geq T_{0}$,

$$
\left|e^{-\int_{s}^{t} a(u) d u}\right| \leq e^{-\alpha(t-s)} .
$$

Therefore,

$$
-\int_{s}^{t} a(u) d u \leq-\alpha(t-s), \quad \frac{1}{t-s} \int_{s}^{t} a(u) d u \geq \alpha .
$$

This implies that $M(a)>0$.

Similarly, one shows that $M(a)<0$ if the latter holds.

This completes the proof.

Using Lemma $4.1 n$ times, we have the following theorem.

THEOREM 4.2. Let the matrix $A(t)$ in (1.6) be real, bounded and continuous such that $a_{i j}=0$ for all $i>j$ and for $1 \leq i \leq n, a_{i i}$ be ergodic. Then (1.6) has a unique bounded solution for every $f \in \mathscr{C}(J)^{n}$ if and only if $M\left(a_{i i}\right) \neq 0,1 \leq i \leq n$. In this case, if $A$ and $f$ are asymptotically almost periodic, then so is the unique bounded solution. Furthermore, if $G(t)$ and $g$ are almost periodic components of $A(t)$ and $f$, respectively, then the equation

$$
\frac{d x}{d t}=G(t) x+g(t)
$$

has a unique almost periodic solution. 
Finally, we give an example to point out that (4.1) (and so (1.1)) may not have a bounded solution if $a$ is not ergodic.

EXAMPLE 4.3. For $n=1,2, \ldots$ and $0 \leq i<n$, let $a_{1}=0, a_{n+1}=a_{n}+n+n^{2}$, and intervals $I_{n}^{i}=\left[a_{n}+i, a_{n}+i+1\right]$. Define a nonnegative, continuous function $g$ on $[0,1]$ such that $g(0)=g(1)=0$ and

$$
\int_{0}^{1} g(t) d t=1
$$

Define the function $\varphi$ on $\mathbb{R}$ by

$$
\varphi(t)= \begin{cases}g\left[t-\left(a_{n}+i\right)\right], & t \in I_{n}^{i} \text { for some } n \text { and some } i, \\ 0, & t \in \mathbb{R}^{+} \backslash \bigcup\left\{I_{n}^{i}: n=1,2, \ldots ; 0 \leq i<n\right\}, \\ \varphi(-t), & t<0 .\end{cases}
$$

The function $\varphi$ is even, bounded, uniformly continuous on $\mathbb{R}$, and

$$
\int_{a_{n}}^{a_{n}+j} \varphi(t) d t=\sum_{i=0}^{j-1} \int_{a_{n}+i}^{a_{n}+i+1} \varphi(t) d t=j, \quad j=1,2, \ldots, n .
$$

It follows from (4.15) that, for $a_{k} \leq T<a_{k+1}$,

$$
\frac{1}{T} \int_{0}^{T} \varphi(t) d t \leq \frac{1}{T} \sum_{n=1}^{k} \int_{a_{n}}^{a_{n}+n} \varphi(t) d t \leq \frac{1}{T} \sum_{n=1}^{k} n \leq \frac{\sum_{n=1}^{k} n}{\sum_{n=1}^{k-1} n^{2}}=\frac{4 k(k+1)}{2(k-1)^{2} k^{2}} \longrightarrow 0 .
$$

If $\varphi$ were ergodic, then

$$
\frac{1}{T} \int_{0}^{T} \varphi(t+s) d t \rightarrow 0 \quad \text { as } T \rightarrow \infty
$$

uniformly in $s \in \mathbb{R}$. However, when $T=j$ and $s=a_{n}$ it follows from (4.15) that

$$
\frac{1}{j} \int_{0}^{j} \varphi\left(t+a_{n}\right) d t=\frac{1}{j} \int_{a_{n}}^{a_{n}+j} \varphi(t) d t=1, \quad j=1,2, \ldots, n .
$$

This is a contradiction. This shows that $\varphi(\cdot)$ is not ergodic.

By symmetry,

$$
\int_{-a_{n}-j}^{-a_{n}} \varphi(t) d t=j, \quad j=1,2, \ldots, n, \quad \lim _{T \rightarrow-\infty} \frac{1}{-T} \int_{T}^{0} \varphi(t) d t=0 .
$$

For any $\alpha \in(-1 / 2,0)$, let $a=\alpha+\varphi$. Then $a$ is not ergodic, $\lim _{t \rightarrow \infty} \int_{0}^{t} a(u) d u / t=$ $\lim _{t \rightarrow-\infty} \int_{t}^{0} a(u) d u /(-t)=\alpha$. In (4.1) let $f=1$. Now we show that (4.1) does not have a bounded solution. In fact, the general solution of (4.1) is

$$
y(t)=e^{\int_{0}^{t} a(u) d u}\left\{C+\int_{0}^{t} e^{-\int_{0}^{s} a(u) d u} d s\right\},
$$

where $C$ is arbitrary. Note $\exp \left\{\int_{0}^{t} a(u) d u\right\} \rightarrow \infty$ as $t \rightarrow-\infty$. Then $y$ is unbounded unless

$$
C+\int_{0}^{t} e^{-\int_{0}^{s} a(u) d u} f(s) d s \rightarrow 0 \quad \text { as } t \rightarrow-\infty .
$$


In this case,

$$
C=-\int_{0}^{-\infty} e^{-\int_{0}^{s} a(u) d u} d s .
$$

Substitute $C$ into (4.20), we get

$$
y(t)=\int_{-\infty}^{t} e^{\int_{s}^{t} a(u) d u} d s .
$$

Let $r=s+a_{n}$,

$$
\begin{aligned}
\left|y\left(-a_{n}\right)\right| & \geq \int_{-a_{n}-n}^{-a_{n}-n+1} e^{\int_{s}^{-a_{n}}[\alpha+\varphi(u)] d u} d s \\
& =\int_{-n}^{-n+1} e^{\int_{r-a_{n}}^{-a_{n}}[\alpha+\varphi(u)] d u} d r \\
& =\int_{-n}^{-n+1} e^{-r \alpha+\int_{r-a_{n}}^{-a_{n}} \varphi(u) d u} d r \\
& \geq \int_{n-1}^{n} e^{n \alpha+(n-1)} d r \geq e^{n / 2-1} \rightarrow \infty .
\end{aligned}
$$

Anyway, $y$ is unbounded.

ACKNOWLEDGEMENT. The work was supported in part by NSFs of China (\#19171019), Leilongjiang Province and HIT.

\section{REFERENCES}

[1] E. Ait Dads, K. Ezzinbi, and O. Arino, Periodic and almost periodic results for some differential equations in Banach spaces, Nonlinear Anal. 31 (1998), no. 1-2, 163-170. MR 98j:34116. Zbl 918.34061.

[2] L. Amerio and G. Prouse, Almost-Periodic Functions and Functional Equations, The University Series in Higher Mathematics, Van Nostrand Reinhold, New York, 1971. MR 43\#819. Zbl 215.15701.

[3] W. Arendt and C. J. K. Batty, Almost periodic solutions of first- and second-order Cauchy problems, J. Differential Equations 137 (1997), no. 2, 363-383. MR 98g:34099. Zbl 879.34046.

[4] B. Basit, Some problems concerning different types of vector valued almost periodic functions, Dissertationes Math. (Rozprawy Mat.) 338 (1995), 26. MR 96d:43007. Zbl 828.43004.

[5] W. A. Coppel, Almost periodic properties of ordinary differential equations, Ann. Mat. Pura Appl. (4) 76 (1967), 27-49. MR 36\#4076. Zbl 153.12301.

[6] _ Dichotomies in Stability Theory, Lecture Notes in Mathematics, vol. 629, SpringerVerlag, New York, 1978. MR 58\#1332. Zbl 376.34001.

[7] C. Corduneanu, Almost Periodic Functions, 2nd ed., Chelsea Publishing Company, New York, 1989, with the collaboration of N. Gheorghiu and V. Barbu, translated from the Romanian by Gitta Berstein and Eugene Tomer. Zbl 672.42008.

[8] W. F. Eberlein, Abstract ergodic theorems and weak almost periodic functions, Trans. Amer. Math. Soc. 67 (1949), 217-240. MR 12,112a. Zbl 034.06404.

[9] A. M. Fink, Almost Periodic Differential Equations, Lecture Notes in Mathematics, vol. 377, Springer-Verlag, New York, 1974. MR 57\#792. Zbl 325.34039.

[10] M. Fréchet, Les fonctions asymptotiquement presque-périodiques, Revue Sci. (Rev. Rose Illus.) 79 (1941), 341-354 (French). MR 7,127e. Zbl 061.16301.

[11]_L Les fonctions asymptotiquement presque-periodiques continues, C. R. Acad. Sci. Paris 213 (1941), 520-522 (French). MR 5,96b. Zbl 026.22102. 
[12] T. Furumochi, Almost periodic solutions of integral equations, Nonlinear Anal. 30 (1997), no. 2, 845-852, Proceedings of the Second World Congress of Nonlinear Analysis, Part 2 (Athens, 1996). MR 98k:45011. Zbl 889.45014.

[13] Y. Hino, S. Murakami, and T. Yoshizawa, Almost periodic solutions of abstract functionaldifferential equations with infinite delay, Nonlinear Anal. 30 (1997), no. 2, 853-864, Proceedings of the Second World Congress of Nonlinear Analysis, Part 2 (Athens, 1996). MR 98k:34113. Zbl 891.34076.

[14] M. N. Islam, Almost periodic solutions of nonlinear integral equations, Nonlinear Anal. 30 (1997), no. 2, 865-869, Proceedings of the Second World Congress of Nonlinear Analysis, Part 2 (Athens, 1996). CMP 1487 667. Zbl 889.45015.

[15] S. Kato, Some remarks on nonlinear ordinary differential equations in a Banach space, Nonlinear Anal. 5 (1981), no. 1, 81-93. MR 81m:34081. Zbl 449.34047.

[16] S. Kato and M. Imai, On the existence of periodic solutions and almost periodic solutions for nonlinear systems, Nonlinear Anal. 24 (1995), no. 8, 1183-1192. MR 96c:34067. Zbl 834.34045.

[17] M. A. Krasnosel'skij, V. S. Burd, and Y. S. Kolesov, Nonlinear Almost Periodic Oscillations, Halsted Press [A division of John Wiley \& Sons], New York, Toronto, Ont.; Israel Program for Scientific Translations, Jerusalem, London, 1973, translated from Russian by A. Libin. Translation edited by D. Louvish. MR 49\#9334. Zbl 287.34038.

[18] B. M. Levitan and V. V. Zhikov, Almost Periodic Functions and Differential Equations, Cambridge University Press, Cambridge, 1982, translated from the Russian by L. W. Longdon. MR 84g:34004. Zbl 499.43005.

[19] J. L. Massera, A criterion for the existence of almost periodic solutions of certain systems of almost periodic differential equations, Bol. Fac. Ingen. Agrimens. Montevideo 6 (1957/58), Also published as Fac. Ingen. Montevideo. Publ. Inst. Mat. Estadist. 3, 1958, pp. 345-349. MR 22\#1709.

[20] W. M. Ruess and W. H. Summers, Asymptotic almost periodicity and motions of semigroups of operators, Linear Algebra Appl. 84 (1986), 335-351. MR 88c:47083. Zbl 616.47047.

[21] _ Minimal sets of almost periodic motions, Math. Ann. 276 (1986), no. 1, 145-158. MR 88a:54092. Zbl 596.54033.

[22] _ Compactness in spaces of vector valued continuous functions and asymptotic almost periodicity, Math. Nachr. 135 (1988), 7-33. MR 89k:46054. Zbl 666.46007.

[23] _ Integration of asymptotically almost periodic functions and weak asymptotic almost periodicity, Dissertationes Math. (Rozprawy Mat.) 279 (1989), 35. MR 90d:46056. Zbl 668.43005.

[24] W. M. Ruess and Q. P. Vu, Asymptotically almost periodic solutions of evolution equations in Banach spaces, J. Differential Equations 122 (1995), no. 2, 282-301. MR 96i:34143. Zbl 837.34067.

[25] T. Yoshizawa, Stability Theory and the Existence of Periodic Solutions and Almost Periodic Solutions, vol. 14, Springer-Verlag, New York, 1975, Applied Mathematical Sciences. MR 57\#6673. Zbl 304.34051.

[26] C. Zhang, Integration of vector-valued pseudo-almost periodic functions, Proc. Amer. Math. Soc. 121 (1994), no. 1, 167-174. MR 94m:43010. Zbl 818.42003.

[27] , Pseudo-almost-periodic solutions of some differential equations, J. Math. Anal. Appl. 181 (1994), no. 1, 62-76. MR 95c:34081. Zbl 796.34029.

[28] _ Vector-valued pseudo almost periodic functions, Czechoslovak Math. J. 47(122) (1997), no. 3, 385-394. MR 98i:42002. Zbl 901.42005.

Chuanyi Zhang: Department of Mathematics, Harbin Institute of Technology, HARBIN, 150001, CHINA

E-mail address: czhang@hope. hit.edu.cn 


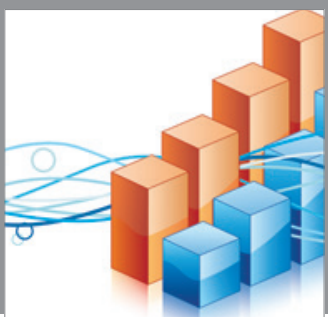

Advances in

Operations Research

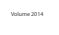

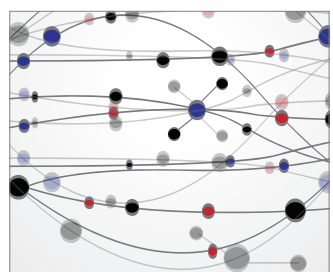

\section{The Scientific} World Journal
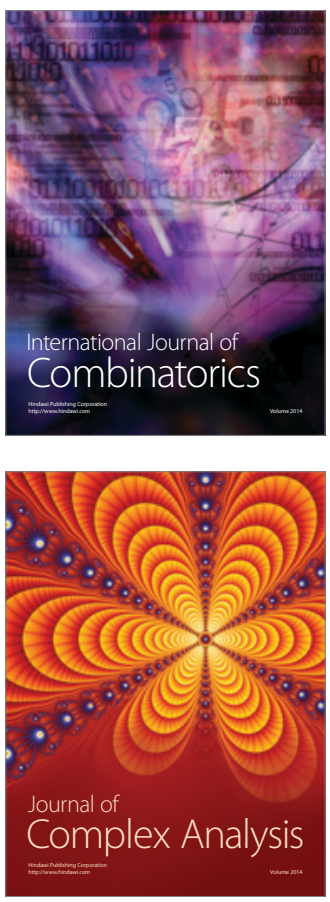

International Journal of

Mathematics and

Mathematical

Sciences
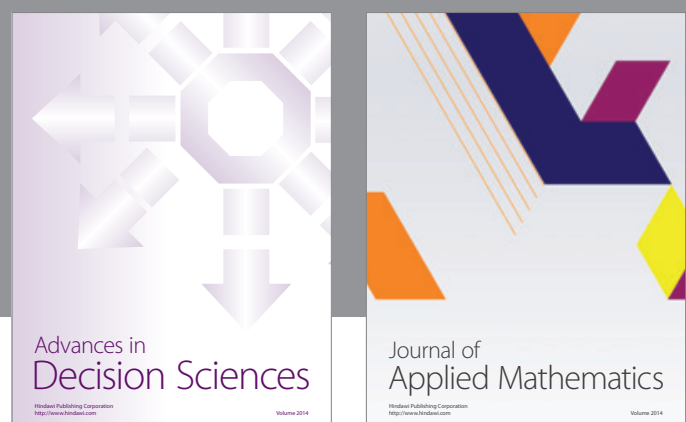

Journal of

Applied Mathematics
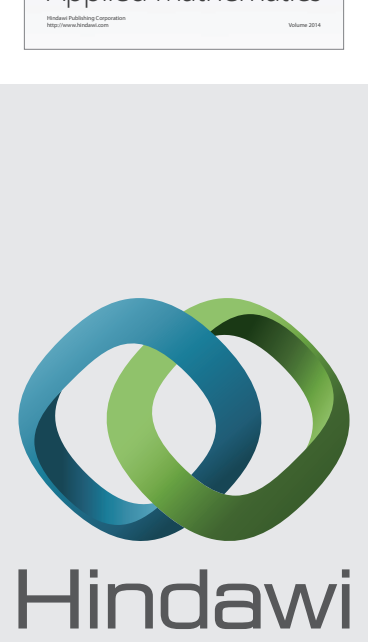

Submit your manuscripts at http://www.hindawi.com
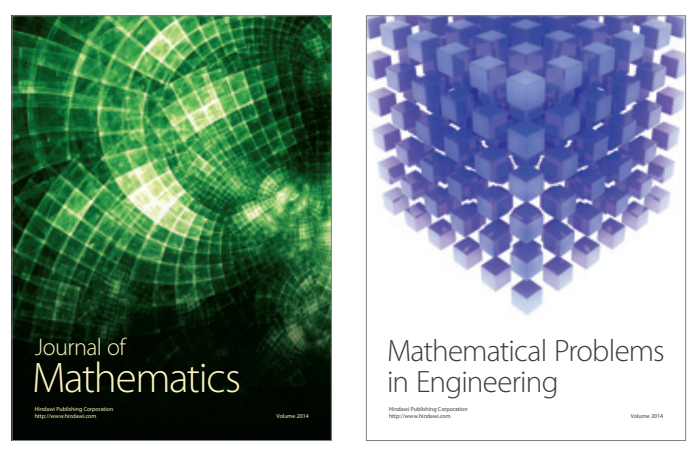

Mathematical Problems in Engineering
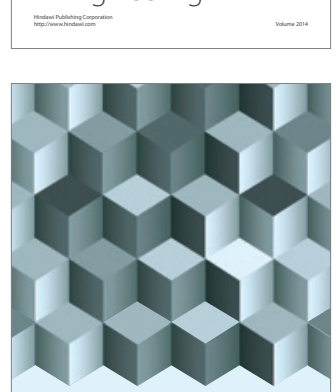

Journal of

Function Spaces
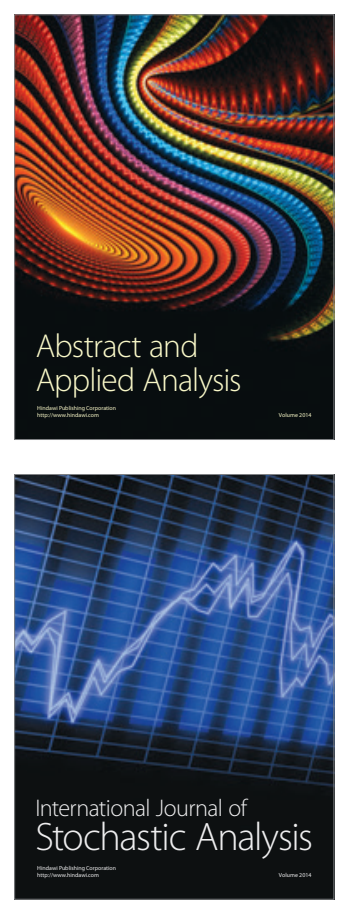

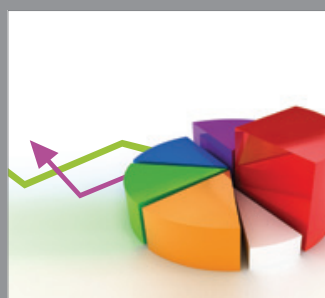

ournal of

Probability and Statistics

Promensencen
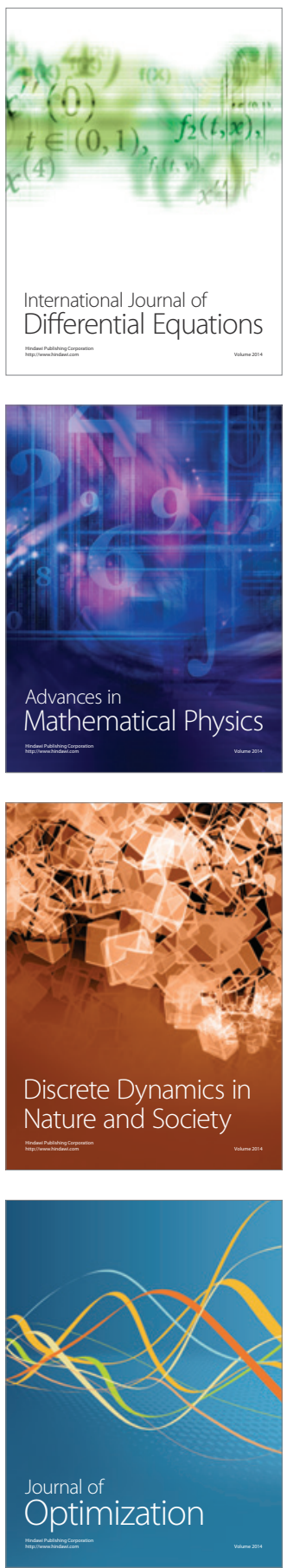